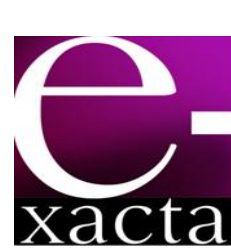

ISSN: 1984-3151

\title{
INFLUÊNCIA DA VARIAÇÃO DA RAZÃO MOLAR ÁLCOOL/ÓLEO E PERCENTUAL DE CATALISADOR NA SÍNTESE DO BIODIESEL A PARTIR DE ÓLEO RESIDUAL
}

\author{
INFLUENCE OF VARIATION OF MOLAR RATION OIL AND ALCOHOL \\ PERCENTAGE OF CATALYST IN THE SYNTHESIS OF BIODIESEL FROM \\ WASTE OIL
}

\author{
Alves $^{3}$ \\ 1 Graduando em Tecnologia em Biocombustíveis. \\ UFPR. Palotina, PR. carlosdo96@gmail.com. \\ 2 Mestrando em Engenharia Industrial. UFBA \\ Professor do Instituto Federal de Educação, Ciência \\ e Tecnologia da Bahia - Campus Irecê. UFBA. \\ (airtonifba@gmail.com) \\ 3 Doutora em Engenharia Industrial. UFBA. 2012. \\ Professora da Universidade Federal do Recôncavo \\ Baiano. Cruz das Almas, BA \\ carinealves60@hotmail.com.
}

Carlos de Jesus de Oliveira ${ }^{1}$; José Airton de Mattos Carneiro Junior ${ }^{2}$; Carine Tondo

Recebido em: 26/02/2015 - Aprovado em: 22/05/2015 - Disponibilizado em: 30/05/2015

RESUMO: O crescente aumento demográfico e industrial juntamente com um desenfreado consumo energético, bem como devido aos impactos ambientais proporcionados pelo uso de combustíveis provenientes de fontes fósseis diminutas, vêm proporcionando uma incessante procura por fontes alternativas de combustíveis. Nesse contexto, os biocombustíveis vêm surgindo como uma alternativa viável em substituição aos combustíveis derivados dos hidrocarbonetos a longo prazo. Entre tais biocombustíveis, podemos citar os ésteres metílicos de ácidos graxos, popularmente conhecidos como biodiesel, o qual tem atraído cada vez mais atenção no intuito de ser utilizado como um combustível alternativo ao diesel. Concomitantemente, elevam-se as expectativas no uso do biodiesel devido ao fato de este ser líquido, neutro e biodegradável, menos poluente comparado ao diesel petroquímico. Além disso, não é tóxico, pois se origina a partir de fontes renováveis, o que leva a uma menor emissão de poluentes durante o processo de combustão. Por conseguinte, devido a sua similaridade ao diesel, pode ser misturado a este usado diretamente em motores do ciclo diesel sem modificações. Dessa forma, o referido trabalho teve como finalidade estudar, através da reação de transesterificação alcalina de óleos residuais utilizando-se um reator de bancada, as impermanentes razão molar e porcentagem de catalisador, tendo como parâmetros fixos da reação a temperatura, agitação e volume do óleo, bem como suas interações. Isso evidencia como resultado que a razão molar álcool / óleo em excesso e porcentagem de catalisador está diretamente na conversão dos triglicerídeos em ésteres metílicos, bem como na qualidade e custo do produto final.

PALAVRAS-CHAVE: Óleo residual. Fontes alternativas. Biodiesel. Biodegradável.

ABSTRACT: The growing population and industrial growth along with a rampant energy consumption, as well as due to environmental impacts provided by the use of fuels from fossil sources miniature, are providing a constant search for alternative sources of fuels. In this context, biofuels are emerging as a viable alternative in place of hydrocarbon-based fuels in the longer term. Among such biofuels, we can cite the methyl esters of fatty acids, popularly known as biodiesel, which have attracted increasing attention in order to use it as an alternative to diesel fuel. Concomitantly, rears up expectations in the use of biodiesel due to the fact of it being liquid, neutral and biodegradable, less polluting compared to petrochemical diesel. Moreover, it is not toxic, as it originates from renewable sources, which leads to lower emissions during the combustion process. Therefore due to its similarity 
to diesel can be mixed with even in different proportions or even used directly in unmodified diesel cycle engines. Thus, this work had as purpose to study through the alkaline transesterification reaction of residual oils using the Workbench reactor impermanent molar ration and percentage of catalyst, with fixed parameters temperature, agitation and reactional oil volume, as well as their interactions. Showing results in the right molar excess oil and alcohol percentage of catalyst is directly in the conversion of triglycerides into methyl esters, as well as the quality and cost of the final product.

KEYWORDS: Residual oil. Alternative source. Biodiesel. Biodegradable.

\section{INTRODUÇÃo}

O incentivo à utilização dos biocombustíveis como combustível alternativo ao diesel no setor de transportes começou mundialmente em 1985, devido à redução da produção de petróleo pela Organização dos Países Exportadores de Petróleo (OPEP), bem como pelo aumento de preços resultante. Nessa época, as matérias-primas para os biocombustíveis advinham de biomassas, tais como: árvores, ervas, microalgas, resíduos do processamento de alimentos, silvicultura, além de resíduos agrícolas e resíduos sólidos urbanos (WU; LIN; CHANG, 2007).

Considerando o crescimento populacional, juntamente com o aumento da demanda energética, nota-se uma incessante procura por novas alternativas de geração de energias renováveis. Esta ação surge em função da representatividade de mais de $80 \%$ das fontes não-renováveis (o carvão, o petróleo bruto e o gás) na demanda energética mundial (AZAD et al., 2015). À vista disso, os ésteres de ácidos graxos, também conhecidos como biodiesel, vêm sendo extensivamente pesquisados devido às suas inúmeras vantagens, sendo um combustível renovável (SCHLEICHER et al., 2009). Neste âmbito, os óleos vegetais apresentam-se como fonte de matérias primas, evidenciando em sua composição cadeias de hidrocarbonetos saturados (triglicerídeos), que consistem de glicerol e ésteres de ácidos graxos. Este último é composto por diferentes comprimentos e números de ligações na cadeia carbônica.
Segundo Kansedo, Lee e Bhatia (2009), atualmente existem quatro diferentes métodos para se reduzir a viscosidade dos óleos vegetais visando a sua utilização como combustível: (a) misturando o óleo vegetal com diesel de petróleo; (b) através dos métodos de pirólise (craqueamento térmico); (c) emulsificação; (d) meio da transesterificação.

No craqueamento térmico, um substrato é reduzido a outro, utilizando-se transferência de calor. Nesta reação pode-se utilizar um catalisador típico ou apenas transferência térmica na ausência de oxigênio ou ar (SAJJADI et al. 2014). Segundo o mesmo autor, nas microemulsões, a viscosidade de óleos vegetais é decrescida através de misturas com solventes, tais como álcoois e/ou compostos anfifílicos iônicos ou não-iônicos. De acordo com Ramachandran et al. (2013), os métodos de pirólise e emulsificação ainda produzem biocombustíveis que acumulam depósito de carbono, combustão incompleta devido ao seu baixo número de cetano. Dessa forma, elevandose a formação de produtos secundários indesejáveis, tais como compostos alifáticos e aromáticos e ácidos carboxílicos.

Diante disso, a transesterificação (ou alcoólise) apresenta-se como um processo simples, quando comparado aos outros métodos, sendo amplamente estudada (SAJJADI et al., 2014). A transesterificação ou alcoólise é uma reação de um óleo ou gordura com um álcool para formação de ésteres e glicerol. $\mathrm{Na}$ reação de transesterificação, há a redução da 
viscosidade dos óleos a um valor semelhante ao diesel normatizado (GHOREISHI; MOEIN, 2013).

Geralmente, na reação de transesterificação, um grande excesso de metanol é usado no intuito de deslocar o equilíbrio para a direita, visando aumentar a formação ésteres (ZHANG et al., 2003).

De acordo com Sánchez et al. (2014), a reação de transesterificação pode ser realizada com a presença de cinco tipos distintos de catalisadores; (i) catalisador homogêneos alcalino considerado muito ativo e barato em comparação aos demais, no entanto, os passos de purificação são mais demorados; (ii) catalisadores homogêneos ácidos são adequados para óleos com um elevado teor de AGL (ácidos graxos livres), porém a velocidade da reação é geralmente mais baixa comparada aos homogêneos alcalinos; (iii) catalisadores heterogêneos alcalinos, os quais, para serem eficazes, requerem elevado volume de álcool em proporção ao volume de óleo utilizado na reação, contudo são mais benéficos ao meio ambiente; (iv) catalisadores heterogêneos ácidos, são menos corrosivos e tóxicos, no entanto possuem preços elevados e apresentam problemas difusionais em comparação aos catalisadores homogêneos; (v) catalisadores enzimáticos possuem facilidade na separação, regeneração e reutilização, mas, por outro lado, apresentam uma baixa taxa de reação e perdem rapidamente a sua atividade. De acordo com Knothe et al. (2006), a utilização dos catalisadores homogêneos alcalinos na reação de transesterificação é justificada pelo elevado rendimento obtido e baixo custo em comparação aos demais catalisadores.

O biodiesel apresenta lubricidade superior, biodegradabilidade, baixa toxicidade, essencialmente ausência de enxofre, alto ponto de fulgor, balanço energético positivo em comparação ao diesel. Assim como contribui na redução do volume de importação do petróleo (MOSER, 2011).
Por conseguinte, os óleos vegetais, bem como as gorduras animais, são as matérias-primas primárias mais utilizadas para a produção de biodiesel, os quais são responsáveis por aproximadamente $75 \%$ do custo de produção (SANTOS et al., 2014).

Deste modo, um possível aumento no uso do biodiesel pode proporcionar uma elevação do custo das matérias-primas tradicionais, interferindo na sua disponibilidade (ARYEEA et al., 2011).

Em outras palavras, a produção de biocombustíveis a partir de fontes de nutrição humana pode causar uma crise alimentar (TALEBIAN-KIAKALAIEH; AMIN; MAZAHERI, 2013).

No entanto, grande parte dos pesquisadores vem concentrando-se na síntese de biodiesel a partir de óleos não comestíveis ou óleos e gorduras residuais (OGRs), tais como óleo de algas, microalgas, pinhãomanso e óleos residuais. Portanto, o uso de óleos residuais alimentares pode contribuir na redução dos custos de produção de biodiesel de $60 \%$ a 90\% (TALEBIAN-KIAKALAIEH; AMIN; MAZAHERI, 2013). O uso de matérias-primas alternativas, de menores custos e não alimentares, é preferível para a síntese do biodiesel (ARYEEA et al., 2011).

Dentre as fontes que não competem com fins alimentares, pode-se citar o óleo de cozinha usado (ou óleo de fritura residual) devido ao fato de ter um valor de compra por litro de 2,5 - 3,0 vezes menor quando comparado aos óleos vegetais virgens (DEMIRBAS, 2009).

Nesse âmbito, o presente trabalho teve como objetivo estudar as variáveis: razão molar álcool/óleo e porcentagem de catalisador, mantendo como parâmetros fixos da reação a temperatura, agitação e volume de óleo utilizado na síntese do biodiesel, através da transesterificação alcalina, a partir de óleos e gorduras residuais obtidas no município de Salvador, Bahia. 


\section{MATERIAIS E MÉtodos}

\subsection{MATERIAIS}

Os óleos residuais utilizados no presente estudo foram adquiridos através de doações de estabelecimentos comerciais e residenciais da cidade de Salvador-BA. Utilizou-se o hidróxido de sódio como catalisador ( $\mathrm{NaOH}$ em micro pérolas, $99 \%$ ), metanol como agente transesterificante e solução de ácido clorídrico $(\mathrm{HCl}$ a $5 \% \mathrm{v} / \mathrm{v})$ para as etapas de purificação. A FIG. 1 apresenta de forma geral as etapas realizadas nesta pesquisa.

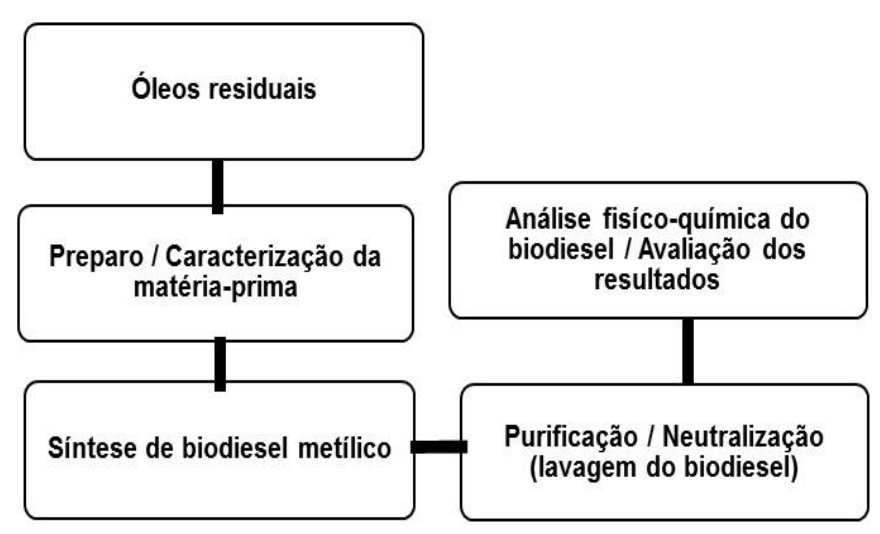

Figura 1 - Fluxograma esquemático das atividades da pesquisa.

\subsection{PrÉ-tratamento da matériA-PRIMA}

Após a coleta, os óleos residuais foram submetidos ao processo de filtragem com papel filtro, visando à remoção de materiais em suspensão e sólidos particulados provenientes do processo de fritura. Segundo Christoff (2006), o sucesso da qualidade do processo de produção de biodiesel, está diretamente ligado à preparação da matéria-prima, como: a) remoção de sólidos suspensos; b) análise do óleo; c) remoção da umidade e; d) preparo do catalisador.

\subsection{CARACTERIZAÇÃO DA MATÉRIA-PRIMA}

Para determinação do índice de acidez utilizou-se o método de titulometria, onde o procedimento consistiu em pesar aproximadamente 2 gramas (g) da matériaprima, dissolvendo-a em $25 \mathrm{~mL}$ de solução éter/álcool (2:1). Na mistura, adicionaram-se 3 gotas do indicador ácido-base fenolftaleína. Em seguida, realizou-se a titulação (em triplicata) usando uma solução titulante de hidróxido de potássio $(\mathrm{KOH})$ $0,1 \mathrm{~mol} \mathrm{~L}^{-1}$, até a persistência da primeira coloração levemente rósea por 30 segundos (RIO et al., 2010).

Em seguida, determinou-se o índice de acidez utilizando a Eq. 1, a qual expressa os resultados em mg de $\mathrm{KOH}$ por g de amostra (Instituto Adolfo Lutz 1985).

$$
I A=V \times N \times 5,61 \div m(1) .
$$

Onde:

IA = Índice de acidez (mg de $\mathrm{KOH} \mathrm{g}^{-1}$ de óleo);

$\mathrm{V}=$ Volume da solução de $\mathrm{KOH}$ gasto na titulação $(\mathrm{mL})$;

$\mathrm{N}=$ Normalidade padronizada da solução de $\mathrm{KOH}$ $\left(\mathrm{mol} \mathrm{L}^{-1}\right)$;

$\mathrm{m}=$ Massa da amostra $(\mathrm{g})$.

\section{Planejamento experimental}

O planejamento experimental teve como intuito obter informações sobre cada um dos fatores estudados, bem como as influências e seus efeitos no processo de síntese da mistura de ésteres, de acordo com estudos realizados por Carvalho, Cabral e AiresBarros (1999).

Foi elaborado planejamento experimental composto por 9 ensaios, realizados em duplicata, utilizando a razão álcool:óleo de 6:1, 8:1, 10:1 e quantidade de catalisador nas proporções de 0,5\%, 0,8 \% e 1,1\%, com tempo de reação de 60 minutos. Esse último parâmetro foi delimitado nessas proporções segundo 
ensaios realizados por Rabu, Janajrehv e Honnery (2013), na síntese de biodiesel. Ainda segundo o autor, o parâmetro tempo varia normalmente de 30 minutos a, cerca de, 120 minutos de reação com concentrações de catalisador de entre 0,1-2\%. Dessa forma, a TAB. 1 evidencia $\circ$ planejamento experimental do referido estudo para a síntese de ésteres metílicos.

Tabela 1

Dados do planejamento experimental das reações

\begin{tabular}{cccc}
\hline Ensaio & Massa do óleo & $\%$ de & Razão molar \\
& $($ g) & cat. & (A/O)
\end{tabular}

\begin{tabular}{l|c|c|c}
\hline 1 & 370,09 & 0,5 & $6: 1$ \\
2 & 370,12 & 0,5 & $8: 1$ \\
3 & 370,60 & 0,5 & $10: 1$ \\
4 & 370,00 & 0,8 & $6: 1$ \\
5 & 370,31 & 0,8 & $8: 1$ \\
6 & 370,07 & 0,8 & $10: 1$ \\
7 & 370,47 & 1,1 & $6: 1$ \\
8 & 370,50 & 1,1 & $8: 1$ \\
9 & 370,60 & 1,1 & $10: 1$ \\
\hline
\end{tabular}

\subsection{SOLUÇÃO CATALISADORA}

O metóxido de sódio $\left(\mathrm{CH}_{3} \mathrm{NaO}\right)$ é caracterizado por uma solução homogênea composta de uma base forte com um álcool de cadeia curta. Geralmente é obtido pela reação de homogeneização do hidróxido de sódio $(\mathrm{NaOH})$ em metanol.

Dessa forma, utilizou-se o metanol devido à sua melhor reatividade, o que implica em menor temperatura e tempo no sistema da reação (LÔBO; FERREIRA; CRUZ, 2009).

O mecanismo da reação pelo qual os catalisadores alcalinos atuam durante a transesterificação é relativamente conhecido. No qual o íon metóxido $\left(\mathrm{CH}_{3} \mathrm{O}^{-}\right)$gerado pela interação do metanol com os sítios básicos do catalisador atacam o grupo carbonila de tri-, di- ou monoglicerídeos levando a produção de ésteres metílicos, além de mono e diglicerídeos liberados no sistema da reação (FERRERO et al., 2015).

Para obtenção do metóxido de sódio, foi pesado o $\mathrm{NaOH}$ variando de 0,$5 ; 0,8$ e 1,1\% em relação à massa de óleo. Em seguida, foi adicionado o volume de metanol às respectivas massas, conforme planejamento experimental, em agitação constante de 900 rpm, até completa dissolução.

Vale ressaltar que a massa do catalisador foi calculada individualmente tendo como base a massa inicial da matéria-prima.

\subsection{Transesterificação ALCALINA}

O mecanismo da reação da transesterificação é evidenciado na FIG. 2.

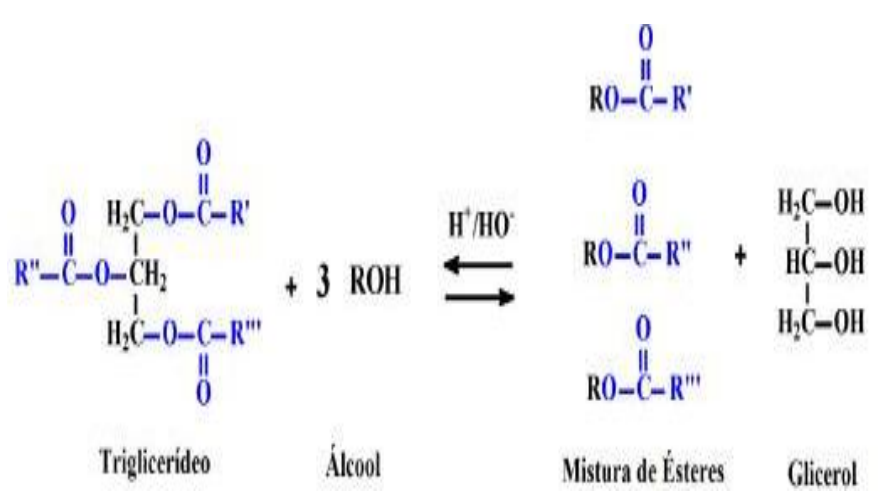

Figura 2 - Mecanismo da reação de transesterificação.

Fonte: Adaptado de Galadima e Muraza (2014).

Conforme Stojković et al. (2014), as principais etapas da síntese de biodiesel são: (a) uma reação de transesterificação; (b) separação das fases brutas do biodiesel e glicerol; (c) separação da águas residuais da lavagem do biodiesel e (d) secagem.

e-xacta, Belo Horizonte, v. 8, n. 1, p. 43-56. (2015). Editora UNIBH. Disponível em: www.unibh.br/revistas/exacta/ 


\section{SínTESE do BIOdieSEL}

\subsection{Sistema dA REAÇÃo E METOdOLOGIA}

Para realização dos ensaios experimentais foi utilizado um reator de bancada de vidro, com capacidade para $1000 \mathrm{~mL}$, equipado com termopar e termômetro digital visando à manutenção das condições de isotermicidade das reações. Possui também sistema de condensação de gases com recirculação de água fria, conforme FIG. 3.

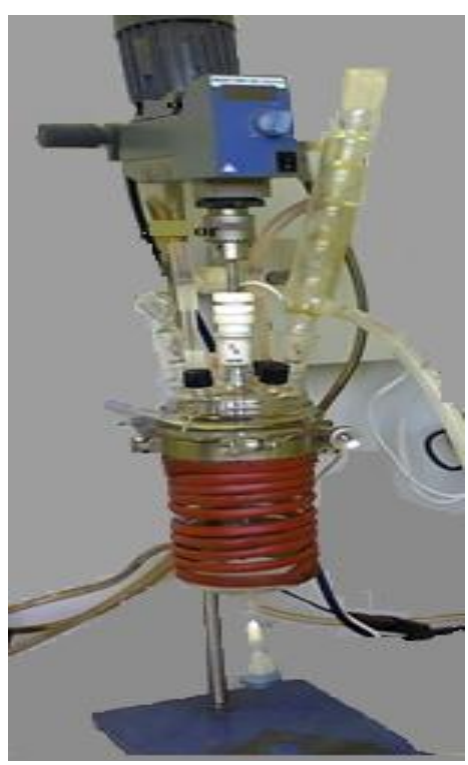

Figura 3 - Reator de bancada utilizado nas reações de transesterificação.

Todos os experimentos foram realizados sobre o mesmo plano da reação, tendo uma temperatura de $55 \pm 0,5{ }^{\circ} \mathrm{C}$, agitação constante de $900 \pm 5 \mathrm{rpm}$ e tempo da reação de 60 minutos. Por outro lado, a razão molar álcool / óleo e a concentração de catalisador foram as variáveis de acordo com planejamento experimental.

A realização dos experimentos foi de forma aleatória, randômica, conforme observado na TAB. 2 .
Tabela 2

Ordem randômica para realização dos experimentos

$\begin{array}{llllllllll}\text { Ensaio } & 5 & 2 & 7 & 9 & 1 & 6 & 4 & 8 & 3\end{array}$

\subsection{Procedimento de síntese do BIOdiesel}

Para a realização da transesterificação alcalina adicionaram-se ao reator as respectivas massas planejadas do óleo residual. Em seguida, ajustou-se o sistema operacional do reator aos parâmetros da reação estipulados no planejamento, tais: temperatura e agitação constante.

Concomitantemente, preparou-se a solução alcoólica de metóxido de sódio pela dissolução da massa do catalisador ao volume de metanol, a agitação constante (500 rpm).

Após estabilizarem-se as condições da reação, acrescentou-se o metóxido de sódio ao sistema da reação, iniciando a reação de transesterificação.

De acordo Fadhil e Ali (2013), o tempo da reação de 60 minutos é ideal, pois reações com durações superiores não demonstraram resultados muito superiores, evitando assim 0 fato da mesma acrescentar um custo extra ao processo.

Decorrido o tempo de reação, transferiu-se a mistura heterogênea para um funil de decantação, permanecendo por 60 minutos, visando a separação das fases da mistura de ésteres brutos e glicerina. A glicerina foi separada e armazenada.

\subsection{PurificaÇão do biodiesel}

A qualidade do biodiesel é de extrema importância quando utilizado na combustão interna dos motores. Assim, se faz necessária a purificação da mistura de ésteres produzida no intuito de remover 
contaminantes, tais como: vestígios residuais de metanol, catalisador, água, sabões, ácidos graxos livres e glicerol, já que tais impurezas comprometem a qualidade do biodiesel final, mesmo quando presentes em baixas quantidades.

Segundo Stojković et al. (2014) e Berrios e Skelton (2008), o processo de purificação do biodiesel tem como objetivo atender às especificações e padrões prescritos pela Agência Nacional do Petróleo, Gás Natural e Biocombustíveis (ANP), órgão responsável pela regulamentação do padrão de qualidade dos biocombustíveis brasileiro.

Nesse âmbito, Berrios et al. (2011) citam a existência de três métodos diferentes para purificação do biodiesel: (a) lavagem com água destilada quente; (b) dissolução em éter de petróleo e lavagem sequencial com água destilada; (c) neutralização com ácido sulfúrico $(1: 1 \mathrm{v} / \mathrm{v})$.

De acordo com os autores, o método mais usual de purificação dos ésteres é através da lavagem com água destilada à temperatura de $50^{\circ} \mathrm{C}$, devido ao fato do glicerol e metanol serem altamente solúveis em água.

Assim, os ésteres produzidos nesse estudo foram lavados com $5 \%$ do volume da matéria-prima inicial, com uma solução de ácido clorídrico $(\mathrm{HCl})$ a $5 \%(\mathrm{v} / \mathrm{v})$, em agitação constante de 150 rpm por 10 minutos. Após esse período, tal mistura permaneceu em repouso por 60 minutos para que ocorresse a decantação.

Em seguida, separou-se a mistura de ésteres da solução aquosa por diferença de densidade, sendo que a solução acidificada tem um maior peso molecular que a mistura de ésteres, permanecendo na parte inferior do funil de separação.

Após hidrólise, a mistura de ésteres bruta foi lavada com 5\% (v/v) de água destilada, com agitação constante de $150 \mathrm{rpm}$ a uma temperatura de $50{ }^{\circ} \mathrm{C}$.
Tal procedimento teve como intuito remover as impurezas e possíveis vestígios de metanol e catalisador. Em seguida, deixou-se a referida mistura em decantação por 60 minutos para uma eficaz separação entre as fases.

Por conseguinte, realizou-se um processo de desumidificação/secagem da mistura de ésteres remanescente. Esta etapa consistiu em aquecer as amostras a temperatura de $105^{\circ} \mathrm{C}$, a $150 \mathrm{rpm}$ por 30 minutos, visando a remoção de partículas de água presentes no meio, por diferença do ponto de ebulição. Sendo em seguida, realizado o ensaio do índice de acidez, em duplicata, por método titulométrico (SILVA, 2010).

\section{ANÁLISE FÍSICO-QUÍMICA DA MISTURA DE ÉSTERES PRODUZIDA}

\subsection{VISCOSIDADE CINEMÁTICA A $40^{\circ} \mathrm{C}$}

A viscosidade cinemática é uma medida da resistência ao fluxo de um líquido, caracterizada pela resistência interna de fluido, esse parâmetro é de extrema importância por estar diretamente relacionada ao processo de injeção de combustível em câmaras de combustão veiculares (MENG; JIA; WANG, 2014).

Dessa forma, visando obter resultados confiáveis sobre a viscosidade cinemática, utilizou-se um viscosímetro capilar de vidro modelo SCHOTT CT 52 , FIG. 4. Onde foram adicionados $10 \mathrm{~mL}$ da amostra no interior do capilar de vidro de 0,63 milímetros (mm).

e-xacta, Belo Horizonte, v. 8, n. 1, p. 43-56. (2015). Editora UNIBH. Disponível em: www.unibh.br/revistas/exacta/ 


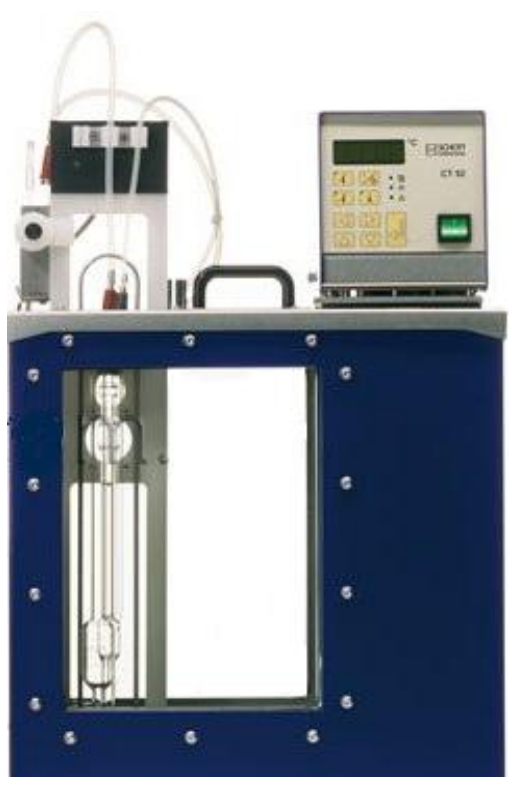

Figura 4 - Viscosímetro capilar, modelo SCHOTT CT 52.

Decorrido o tempo de deslocamento do fluido no capilar, converteu-se o tempo de minutos para segundos, multiplicou-se pela constante do aparelho, fornecida a partir de calibrações pelo fabricante. Para cálculo final da viscosidade cinemática foi utilizada a Eq. 2.

$$
\mathrm{V}=\mathrm{K} \cdot(\mathrm{t}-\vartheta)
$$

Onde:

$\mathrm{V}=$ Viscosidade cinemática $\left(\mathrm{mm}^{2} \mathrm{~s}^{-1}\right.$ ou cSt $)$;

$\mathrm{K}=$ Constante do capilar;

$\mathrm{t}=$ tempo gasto $(\mathrm{s})$;

$\vartheta=$ Correlação da energia cinemática.

A viscosidade é uma característica importante nos combustíveis, pois está relacionada ao fluxo e atomização de combustível líquido na câmara de combustível. Devido a isso, elevadas viscosidades proporcionar uma combustão incompleta, contribuindo para formação deposição de carbono sobre bicos injetores e válvulas (KEERA et al., 2011).

\subsection{MASSA ESPECÍfICA A $20^{\circ} \mathrm{C}$ OU DENSIDADE}

A densidade de uma substância é definida como a massa por unidade de volume (VERDUZCO, 2013). Segundo o mesmo autor, a densidade é uma propriedade física que pode ser usada para calcular o volume preciso de combustível necessário para o fornecimento de uma combustão adequada.

De acordo com Meng, Jia e Wang (2013), a densidade é um parâmetro significativo nos combustíveis líquidos, correlacionada ao número de cetano, valor de aquecimento e viscosidade, dessa forma influenciando diretamente no processo de injeção do combustível na câmara de combustão. Tendo em vista obter resultados esclarecedores sobre a massa específica, utilizou-se densímetro digital ANTON PAAR, mod. DMA $35 \mathrm{~N}$. Onde se coletou aproximadamente $1 \mathrm{~mL}$ de cada amostra as quais foram aspiradas por meio de tubo capilar flexível para o interior do aparelho, obtendo-se assim um resultado confiável relacionado a massa específica a $20^{\circ} \mathrm{C}$.

\section{RESUltados E discussões}

Visando garantir a qualidade do biodiesel, foram estabelecidos padrões de qualidade objetivando fixar teores limites dos interferentes que possam vir a prejudicar a qualidade da queima, bem como o desempenho, integridade do motor, segurança no transporte e manuseio.

Independente da rota tecnológica de produção, a aceitação do biodiesel no mercado precisa ser assegurada, e para isso é indispensável que esse produto esteja dentro das especificações internacionais aceitas para seu uso, conforme Oliveira et al., 2014.

Embora os parâmetros de qualidade do biodiesel tenham origem na normatização do diesel mineral, os mesmos fornecem resultados esclarecedores quanto à 
qualidade da mistura de ésteres produzida. Dessa forma, pode ser observada na TAB. 3 a caracterização da mistura de ésteres produzida laboratorialmente.

Tabela 3

Resultados da caracterização da mistura de ésteres produzida em laboratório

\begin{tabular}{c|c|c|c}
\hline \multicolumn{5}{c}{$\begin{array}{c}\text { Índice de } \\
\text { Reação }\end{array}$} & $\begin{array}{c}\text { Viscosidez } \\
\text { cinemática } \\
\text { a } 40^{\circ} \mathrm{C}\end{array}$ & $\begin{array}{c}\text { Massa } \\
\text { específica a } \\
\text { (mg KOH })\end{array}$ \\
$\begin{array}{c}\mathrm{mm}^{2} \mathrm{~s}^{-1} \text { ou } \\
\text { cSt })\end{array}$ & $20^{\circ} \mathrm{C}\left(\mathrm{kg} \mathrm{m}^{-3}\right)$ \\
\hline 1 & 0,834 & 4,56 & 884,4 \\
3 & 0,839 & 4,79 & 886,0 \\
4 & 0,838 & 4,13 & 883,4 \\
5 & 1,395 & 4,12 & 883,0 \\
6 & 1,022 & 4,85 & 886,2 \\
7 & 0,837 & 4,05 & 883,2 \\
8 & 1,397 & 4,09 & 882,9 \\
9 & 1,114 & 4,53 & 884,3 \\
\hline
\end{tabular}

Vale ressaltar que o índice de acidez do óleo residual bruto variou de 1,96 a $1,98 \mathrm{mg}$ de $\mathrm{KOH}$ por grama de óleo.

\subsection{Volume DA MISTURA DE ÉSTERES PRODUZIDA}

A FIG. 5 apresenta a relação de fase glicerinosa e fase éster obtida da reação de transesterificação. Assim, evidencia os resultados comparativos entre 0 volume da mistura de ésteres produzida e a porção de glicerina gerada.

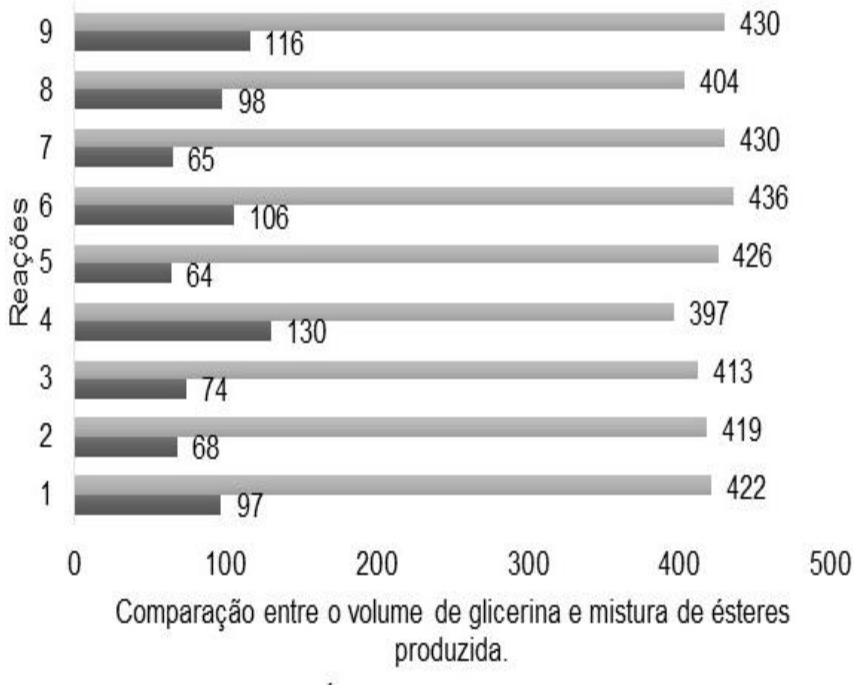

\Volume da Mistura de Ésteres $(\mathrm{mL}) \quad$ volume de Glicerina $(\mathrm{mL})$

Figura 5 - Comparação entre o volume de glicerina e mistura de ésteres produzida.

Pode-se observar que os maiores volumes das misturas de ésteres foram obtidos nos experimentos 6 e 9, com $436 \mathrm{~mL}$ e $430 \mathrm{~mL}$, respectivamente. Tal fato pode ser justificado devido ambos os ensaios partirem de uma razão molar de álcool/óleo de 10:1, uma vez que geralmente um excesso de álcool é utilizado visando deslocar o equilíbrio da reação para direita, contribuindo para a formação de produtos, sendo neste caso a mistura de ésteres. Em conformidade com Ramos et al. (2011), a razão molar álcool:óleo estequiométrica na transesterificação é de 3:1 (três mols de álcool para um mol de óleo), porém, um amplo excesso de álcool, usualmente $6: 1$ ou 12:1, é utilizado para deslocar o equilíbrio químico a fim de maximizar a produção de ésteres graxos.

Conforme literatura, proporções molares mais elevadas resultam em uma maior conversão do triglicerídeo em éster, em menor tempo (MA; HANNA, 1999). Por outro lado, o menor volume da mistura de ésteres produzida neste trabalho foi evidenciado no ensaio de número 4 (397 $\mathrm{mL})$, provavelmente a redução do volume foi ocasionada em decorrência de reações secundárias de saponificação e, devido a correlação do elevado índice de acidez da matéria- 
prima e uma menor proporção molar álcool/óleo (6:1) em comparação aos demais experimentos.

Nesse mesmo âmbito, Agarwal et al. (2012) citaram que uma maior massa de catalisador mantendo um mesmo volume de óleo e metanol na síntese de biodiesel, proporciona uma solução catalisadora mais concentrada, na qual resulta em um déficit na homogeneidade da mistura na mesma velocidade de agitação. Consequentemente, acarretando no acúmulo de catalisador devido à resistência da transferência mássica. Ainda, o aumento da quantidade de catalisador, torna a mistura mais viscosa, acarretando a um problema de mistura, além de possíveis formações de sabões devido a reações secundárias de saponificação.

\section{2 ÍNDICE DE ACIDEZ DAS MISTURAS DE ÉSTERES}

O índice de acidez é um parâmetro de qualidade de óleos e gorduras, que reflete a quantidade de ácidos graxos livres presentes nas amostras. Nesse âmbito, a FIG. 6, apresenta o índice de acidez da mistura de ésteres produzida.

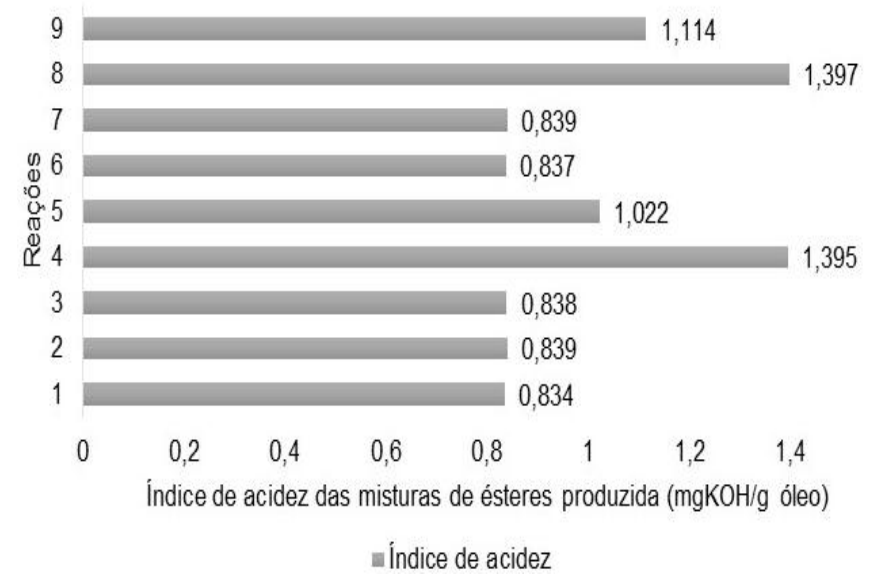

Figura 6 - Resultados do índice de acidez por amostra.
De acordo com a FIG. 6, nota-se que todas as amostras analisadas evidenciaram um índice de acidez superior ao limite máximo $\left(0,5 \mathrm{mg} \mathrm{KOH} \mathrm{g}^{-1}\right.$ óleo) estabelecido pela ANP. O elevado índice de acidez encontrado em todas as mistura de ésteres produzida neste trabalho, relata a ineficiência no processo de purificação adotado e/ou influência do avançado estado degradativo dos óleos residuais utilizados como matéria-prima.

Nesse mesmo contexto, Canesin et al. (2014) afirma que $80 \%$ das amostras de biodiesel tendo como matéria-prima óleos animais e óleos residuais, não satisfizeram o limite máximo de $0,5 \mathrm{mg}^{\mathrm{de} \mathrm{KOH} \mathrm{g}}{ }^{-1}$ óleo estabelecido pela norma ASTM D664. Porém, os experimentos de maiores volumes da mistura de ésteres (6, 7 e 9), houve uma notável diferença no índice de acidez no produto final, sendo que no experimento 9, com $430 \mathrm{~mL}$ de biodiesel obteve-se acidez de $1,1 \mathrm{mg} \mathrm{KOH} \mathrm{g}^{-1}$ óleo. Por outro lado, o experimento 6 , com $436 \mathrm{~mL}$ de biodiesel, apresentou um índice de acidez de 0,8 $\mathrm{mg}$ de $\mathrm{KOH}$, fato que pode ser explicado devido ao diferente percentual de catalisador utilizado no experimento 9 , cerca de 1,1\% e no experimento 6 utilizou-se $0,8 \%$ ambos com a mesma razão molar álcool/óleo (10:1), temperatura e agitação. E, segundo Sánchez et al. (2014), a adição de excesso de catalisador no meio da reação não proporciona um rendimento superior dos ésteres metílicos.

Com o mesmo intuito, Leung e Guo (2006) examinaram o rendimento do biodiesel sob os casos de excesso de concentrações de $\mathrm{NaOH}$ (massa maior que $1,0 \%$ ) concluindo que um possível aumento na concentração de $\mathrm{NaOH}$ de 1,0 até $1,6 \%$ em peso, proporcionou uma diminuição no rendimento biodiesel produzido (com uma pureza de cerca de $98 \%$ em peso) de $85 \%$ para $65 \%$ em peso. Além de grandes quantidades de sabão com o excesso de catalisador, $\mathrm{NaOH}$, adicionado foram evidenciado. 
Por outro lado a hipótese da lavagem com ácido pode ter contribuído para um possível aumento no índice de acidez, nos experimentos 4 e 8 , os quais obtiveram os maiores índice de acidez do biodiesel.

Dessa forma, necessitando de um aumento no número de lavagem com água a $50^{\circ} \mathrm{C}$, seria a opção mais viável, visando à purificação e adequação da mistura de ésteres aos padrões de qualidades estabelecidos pela ANP.

De acordo com Fadhil, Dheyab e Abdul-Qader (2012), tal método utiliza um volume excessivo de água em agitação suave para remoção de impurezas, tais como glicerol, metanol e a base (catalisador) que não reagiram. Esta técnica baseia-se na afinidade de compostos polares com água.

Segundo Grangeiro (2009), considerando os métodos tradicionais de lavagem, necessita-se de 3 litros de água para cada litro de biodiesel purificado. Além disso, as águas resultantes desse processo de purificação caracterizam-se como inadequadas quimicamente e biologicamente para serem lançadas a qualquer corpo hídrico de acordo com a legislação brasileira, em particular a resolução № 430, de 13 de maio de 2011 - CONAMA (2011).

\subsection{MASSA ESPECÍFICA A $20^{\circ} \mathrm{C}$ DAS MISTURAS DE ÉSTERES}

A FIG. 7 compara os resultados da massa específica a $20{ }^{\circ} \mathrm{C}$ e a viscosidade cinemática da mistura de ésteres produzida via transesterificação alcalina.

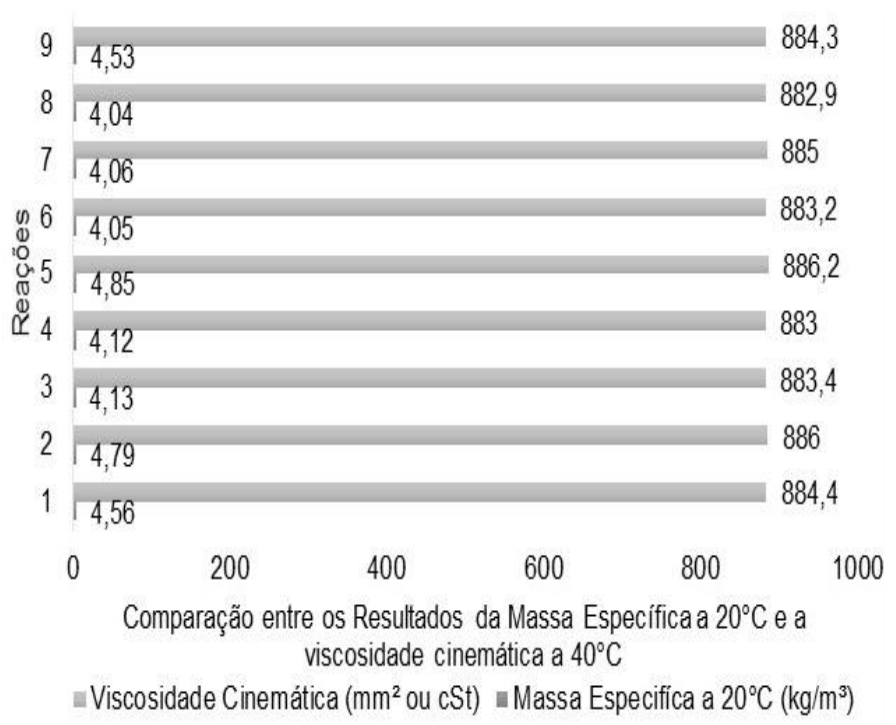

Figura 7 - Comparação dos resultados das análises entre a massa específica e viscosidade cinemática $40^{\circ} \mathrm{C}$.

Vale ressaltar que o óleo residual apresentou densidade de média de $889,0 \mathrm{~kg} \mathrm{~m}^{-3}$.

Dessa maneira, a FIG. 7 evidencia a análise dos resultados médios da viscosidade cinemática a $40^{\circ} \mathrm{C}$ dos experimentos. Nota-se que os dados médios obtidos ficaram entre $4,05-4,85 \mathrm{~mm}^{2}$ ou cSt, ou seja, dentro do limite estabelecido pela norma da Agencia Nacional do Petróleo, Gás natural e Biocombustíveis (ANP), onde esse parâmetro estabelece um limite máximo entre $3,0-6,0 \mathrm{~mm}^{2}$ ou cSt. Por conseguinte resultados próximos aos obtidos por Christoff (2006), em pesquisas de síntese de ésteres metílicos tendo como matéria-prima os óleos residuais.

Observa-se também na FIG. 7 os resultados da análise da massa específica a $20^{\circ} \mathrm{C}$ ou densidade da mistura de ésteres produzida, as quais ficaram entre 882,9 - 886,2 $\mathrm{kg} \mathrm{m}^{-3}$, enquadrando-se ao padrão de qualidade exigido pela ANP a qual limita a densidade do em $850-900 \mathrm{~kg} \mathrm{~m}^{-3}$. 


\section{CONSIDERAÇõES FINAIS}

Pode-se observar a viabilidade da produção de ésteres metílicos via transesterificação alcalina, tendo como matéria-prima os óleos e gorduras residuais provenientes do processo de fritura de alimentos. Nesse contexto, foram obtidos dados científicos que poderão ser utilizados em trabalhos futuros, assim como, para avaliação da conformidade com o padrão estabelecido pela agência Nacional de Petróleo, Gás Natural e Biocombustíveis - ANP.

Foram obtidos melhores resultados em relação ao maior volume e menor viscosidade cinemática a $40{ }^{\circ} \mathrm{C}$, bem como, menor massa especifica a $20{ }^{\circ} \mathrm{C}$ das misturas de ésteres produzidas, obtidos quando utilizado uma razão molar álcool/óleo (10:1), 0,8 \% de catalisador básico, tendo como parâmetros fixos temperatura de $55 \pm 0,5^{\circ} \mathrm{C}$, agitação constante em $900 \pm 5 \mathrm{rpm}$.

Por outro lado, verificou-se que a razão molar álcool/óleo em excesso, bem como a porcentagem de catalisador, não está diretamente ligada apenas com a uma melhor conversão dos triglicerídeos em ésteres metílicos, mas também na qualidade do produto final do mesmo modo que tais parâmetros podem interferir diretamente na redução do tempo da reação de produção.

\section{REFERÊNCIAS}

AGARWAL, M. et al. Study of catalytic behavior of $\mathrm{KOH}$ as homogeneous and heterogeneous catalyst for biodiesel production. Journal of the Taiwan Institute of Chemical Engineers, v.43, p.89-94. 2012.

ARYEEA, N. A. A. et al. Enzymatic transesterification of fats and oils from animal discards to fatty acid ethyl esters for potential fuel use. Biomass and Bioenergy, v35, p.4149-4157. 2011.

AZAD, A. K. et al. Prospect of biofuels as an alternative transport fuel in Australia. Renewable and Sustainable Energy Reviews, v. 43, p.331-351, 2015.

BERRIOS, M. et al. Purification of biodiesel from used cooking oils: Purification of biodiesel from used cooking oils. Applied Energy, v.88, p.3625-3631. 2011.

BERRIOS, M.; SKELTON, R. L. Comparison of purification methods for biodiesel. Chemical Engineering Journal, v.144, p.459-465. 2008.

CANESIN, E. A. et al. Characterization of residual oils for biodiesel production. Electronic Journal of Biotechnology, v.17, p.39-45, 2014.

CARVALHO, C. M. L., CABRAL, J. M. S.; AIRESBARROS, M. R. Cutinase stability in AOT reversed micelles: system optimization using the factorial design methodology. Enzyme and Microbial Technology, v.24, p.569-576, 1999.
CHRISTOFF, P. Produção de biodiesel a partir do óleo residual de fritura comercial: estudo de caso: Guaratuba, litoral paranaense. 2006. $82 \mathrm{f}$. Dissertação (Mestrado) - Curso de área Desenvolvimento de Tecnologia, Instituto de Tecnologia Para O Desenvolvimento - LACTEC e Instituto de Engenharia do Paraná, Curitiba, 2006.

CONAMA - CONSELHO NACIONAL DO MEIO AMBIENTE. RESOLUÇÃO N 430: Condições, parâmetros, padrões e diretrizes para gestão do lançamento de efluentes em corpos de água receptores: Ministério do Meio Ambiente, 2011. Disponível em:

<http://www.mma.gov.br/port/conama/res/res11/propre sol lanceflue 30e31mar11.pdf $>$. Acesso em: $10 \mathrm{abr}$. 2015.

DEMIRBAS, A. Biodiesel from waste cooking oil via base-catalytic and supercritical methanol transesterification. Energy Conversion and Management, v.50, p.923-927, 2009.

FADHIL, A. B.; ALI, L. H. Alkaline-catalyzed transesterification of silurus triostegus heckel fish oil: Optimization of transesterification parameters. Renewable Energy, v.60, n.34, p.481488, 2013.

FADHIL, A. B.; DHEYAB, M. M.; ABDUL-QADER, A. $Y$. Purification of biodiesel using activated carbons produced from spent tea waste. Journal of the Association of Arab Universities for Basic and Applied Sciences, v. 11, p.45-49, 2012. 
FERRERO, G. O. et al. Glycerol-enriched heterogeneous catalyst for biodiesel production from soybean oil and waste frying oil. Energy Conversion and Management, v. 89, p.665-671, 2015.

GALADIMA, A.; MURAZA, O. Biodiesel production from algae by using heterogeneous catalysts: A critical review. Energy, v. 78, p. 72-83, 2014.

GHOREISHI, S. M; MOEIN, P. Biodiesel synthesis from waste vegetable oil via transesterification reaction in supercritical methanol. The Journal of

Supercritical Fluids, v. 76, p.24-31, 2013.

GRANGEIRO, R. V. T. Caracterização da água de lavagem proveniente da purificação do

biodiesel. 2009. 40 f. Dissertação (Mestrado) - Curso de Química, Universidade Federal da Paraíba, João Pessoa - PB, 2009.

INSTITUTO ADOLFO LUTZ. Normas analíticas do Instituto Adolfo Lutz: Métodos Químicos e Físicos para Análise de Alimentos. 3a edição, São Paulo: IMESP, 1985. P.245-246.

KANSEDO, J.; LEE, K. T.; BHATIA, S. Cerbera odollam (sea mango) oil as a promising non-edible feedstock for biodiesel production. Fuel, v.88, n.6, p.1148-1150, 2009.

KEERA, S. T.; SABAGH, S. M. E; TAMAN, A. R. Transesterification of vegetable oil to biodiesel fuel using alkaline catalyst. Fuel, v. 90, p.42-47, 2011.

KNOTHE, G.; GERPEN, J. V.; KRAHL, J.; RAMOS, L. P. Manual de Biodiesel. São Paulo: Edgard Blucher, 2006. 340 p.

LEUNG, D. Y. C.; GUO, Y. Transesterification of neat and used frying oil: Optimization for biodiesel production. Fuel Processing Technology, v.87, p.883-890, 2006.

LÔBO, I. P.; FERREIRA, S. L. C.; CRUZ, R. S. BIODIESEL: Parâmetros de qualidade e métodos analíticos. Quim. Nova, v.32, n.6, p.1596-1608, 2009.

MA, F.; HANNA, M. A. Biodiesel production: a review. Bioresource Tecnology, 70, p.1-15, 1999.

MENG, X.; JIA, M.; WANG, T. Neural network prediction of biodiesel kinematic viscosity at 313 K. Fuel, v.121, p.133-140, 2014.

MENG, X.; JIA, M.; WANG, T. Predicting biodiesel densities over a wide temperature range up to 523 K. Fuel, v.111, p.216-222, 2013.
MOSER, B. R. Influence of extended storage on fuel properties of methyl esters prepared from canola, palm, soybean and sunflower oils. Renewable Energy, v. 36, p.1221-1226, 2011.

OLIVEIRA, R. S. et al. Avaliação da ação antioxidante de produtos naturais no biodiesel b100 (glycine max). Rev. da Escola de Eng. da UFF (ENGEVISTA), v.16, n.3, p.410-419, 2014.

RABU, R. A.; JANAJREH, I.; HONNERY, D. Transesterification of waste cooking oil: Process optimization and conversion rate evaluation. Energy Conversion and Management, v. 65, p.764-769, 2013.

RAMACHANDRAN, K. et al. Recent developments for biodiesel production by ultrasonic assist transesterification using different heterogeneous catalyst: A review. Renewable and Sustainable Energy Reviews, v. 22, p.410-418, 2013.

RAMOS, L. P. et al. Tecnologias de Produção de Biodiesel. Revista Virtual de Quimica, v. 3, n. 5, p.385-405, 2011.

SAJJADI, B.; AZIZ, A. R. A.; IBRAHIM, S. Investigation, modelling and reviewing the effective parameters in microwave-assisted transesterification. Renewable and Sustainable Energy Reviews, v.37, p.762-777, 2014.

SÁNCHEZ, M. et al. Biodiesel production optimization using YAl2O3 based catalysts. Energy, v.73, p.661669, 2014.

SANTOS, A. G. D. et al. Kinetic study and thermoxidative degradation of palm oil and biodiesel. Thermochemica Acta, v. 592, p.18-22, 2014.

SCHLEICHER, T. et al. Microbiological stability of biodiesel-diesel-mixtures. Bioresource Technology, v. 100, p.724-730, 2009.

SILVA, J. B. F. Produção de biodíesel etílico de óleos e gorduras residuais (OGR) em reator químico de baixo custo. 2010. 57 f. Dissertação (Mestrado) - Curso de Programa de Pós-Graduação em Engenharia Mecânica e de Materiais - (PPGEM), Universidade Tecnológica Federal do Paraná Campus de Curitiba, Manaus, 2010.

STOJKOVIĆ, I. J. et al. Purification technologies for crude biodiesel obtained by alkali-catalyzed transesterification. Renewable and Sustainable Energy Reviews, v.32, p.1-15, 2014. 
TALEBIAN-KIAKALAIEH, A.; AMIN, N. A. S.; MAZAHERI, $H$. A review on novel processes of biodiesel production from waste cooking oil. Applied Energy, v. 104, p.683-710, 2013.

VERDUZCO, L. F. R. Density and viscosity of biodiesel as a function of temperature: Empirical models. Renewable and Sustainable Energy

Reviews, v.19, p.652-665, 2013.
WU, Y. G.; LIN, Y.; CHANG, C. Combustion characteristics of fatty acid methyl esters derived from recycled cooking oil. Fuel, v.86, p.2810-2816, 2007.

ZHANG, Y. et al. Biodiesel production from waste cooking oil: 1. Process design and technological assessment. Bioresource Technology, v. 88, p.1-16, 2003. 